\title{
An Assessment of the Precise Products on Static Precise Point Positioning using Multi-Constellation GNSS
}

\author{
Jareer Mohammed ${ }^{1,2}$ \\ ${ }^{1}$ College of Engineering, University of Wasit \\ Wasit, Iraq \\ jareermohammed@uowaist.edu.iq \\ Richard M. Bingley ${ }^{3}$ \\ ${ }^{3}$ NERC British Isles Continuous GNSS Facility (BIGF), \\ Nottingham Geospatial Institute, The University of \\ Nottingham, UK Richard.Bingley@nottingham.ac.uk
}

\begin{abstract}
Precise point positioning (PPP) is highly dependent on the precise ephemerides and satellite clock products that are used. Different ephemeris and clock products are available from a variety of different organizations. The aim of this paper is to assess the achievable static positioning accuracy and precision when using different precise ephemerides from three analysis centres Natural Resources Canada (EMX), European Space Agency (ESA) and GeoForschungsZentrum (GFZ), using GPS alone, GLONASS alone, and GPS and GLONASS combined. It will be shown in this paper that the precise products are significantly affected by the time-base of the reference stations, and that this is propagated through to all the estimated satellite clocks. In order to overcome the combined biases in the estimated satellite clock, in the PPP processing, these clocks errors need to be handled with an appropriate variation in the estimated receiver clock. It will also be shown that the precise coordinates of the satellites differ between the analysis centres, and this affects the PPP position estimation at the millimetre level. However, all those products will be shown to result in the same level of precision for all coordinate components and are equivalent to the horizontal precision from a Global Double Difference (GDD) solution. For the horizontal coordinate component, the level of agreement between the PPP solutions, and with the GDD solution, is at the millimetre level. There is a notable, but small, bias in the north coordinate components of the PPP solutions, from the corresponding north component of the GDD solutions. It is shown that this difference is due to the different strategy adopted for the GDD and PPP solutions, with PPP being more affected by the changing satellite systems. The precision of the heights of the receiver sites will be shown to be almost the same across all the PPP scenarios, with all three products. Finally, it will be concluded that accuracy of the height component is system dependent and is related to the behaviour of antenna phase centre with the different constellation type.
\end{abstract}

Keywords; GNSS, GPS, GLONASS, Precise Point Positioning;

\section{INTRODUCTION}

The Precise Point Positioning (PPP) technique [1] has now developed to the level of maturity to allow it to provide daily

\author{
Terry Moore ${ }^{2}$, Chris Hill ${ }^{2}$ \\ ${ }^{2}$ Nottingham Geospatial Institute (NGI), the University of \\ Nottingham \\ Nottingham, United Kingdom \\ Terry.Moore@nottingham.ac.uk
}

solution position estimates with millimetric level repeatability and accuracy for static points [2], and has been shown to be a viable alternative method to the 'conventional' doubledifference (DD) technique. However, the coordinates of positions determined by PPP are highly dependent on the chosen precise ephemeris and satellite clock products. This represents a key factor when comparing different PPP solutions. Gao and Chen [3] assessed the performance of PPP GPS using real-time precise orbit and clock corrections; they compare the receiver clock offsets with the reference clock at AMC2 stations. However, they did not investigate the effect of the system time offsets on the satellites clocks nor the impact these had on the PPP solutions. Cai and Gao [4] investigated the time offsets between GPS and GLONASS in PPP solutions with a suggestion that the difference between the receiver clock offsets for GPS and GLONASS represents the time difference between the two constellations. Reussner and Wanninger [5] used European Space Operations Centre (ESOC) products for assessing PPP with the assessment of inter-frequency biases, without an investigation to the different products. In addition, even though Cai and Gao [6] analyzed the effect of using Information Analytical Center(IAC) and ESA/ESOC products on the position estimation, using a 4 hour dataset from only one station $\mathrm{OHI} 3$, this is a very short sample from which to attempt to evaluate the effect of products on position estimation. They also did not discuss the difference between the satellite orbits or precise clock products from the two analysis centres. Guo, et al. [7] made a quality assessment of the precise orbit and clock products for the emerging Galileo, BeiDou and QZSS system provided by the Multi-GNSS Experiment(MGEX) over two years. However, their focus was on the upcoming constellations without an evaluation to the available fully operational constellations (GPS and GLONASS); and furthermore, they did not mention the effects of reference station time-base. It is notable that there is no comparison of the effect on the time reference on the products and the resultant effect on the precise satellites orbit, and their effect on the estimated coordinate components from PPP. Hence, this paper has four key aims: 
- Investigate the effect of the time-base of the reference stations on the satellite clock products and how this can be handled in the PPP processing strategy.

- Investigate the corresponding effect on the final precise orbits, from different Analysis Centres (AC) (Natural Resources Canada (EMX), European Space Agency (ESA) and GeoForschungsZentrum (GFZ)), and the subsequent effect on the estimated PPP position components.

- Assess the achievable accuracy and precision, from static PPP processing, when using different precise ephemerides from the three ACs, using GPS only (PPP GPS) GLONASS only (PPP GLO) and GPS plus GLONASS (PPP GPS+GLO).

- Analyses the evident biases between the PPP solutions when compared to Global Double Difference (GDD) solutions.

\section{Methodology}

\section{A. PPP Daily Solution Methodology}

All of the PPP daily solutions presented in this paper were processed using the POINT software, which was developed as part of the iNsight project (www.insight-gnss.org)[8]. The POINT software is programmed in $\mathrm{C}++$, and its core is the extended Kalman filter (EKF), as presented in Feng, et al. [9].

Undifferenced observations were used for each PPP daily solution using general observation equations for the code and phase as follows:

$$
\begin{gathered}
P_{F}^{i}=e^{i}+c \delta_{r_{c o d e}}-c \delta^{i}+\frac{I^{i}}{f_{F}^{2}}+\frac{S^{i}}{f_{F}^{3}}+T^{i} \\
+M_{P F}^{i}+Q_{P F}^{i}+\operatorname{bias}_{P, F}-\operatorname{bias}_{P, F}^{i}
\end{gathered}
$$

For the carrier phase $(\mathrm{m})$ :

$$
\begin{gathered}
L_{F}^{i}=e^{i}+c \delta_{r_{p h a s e}}-c \delta^{i}-\frac{I^{i}}{f_{F}^{2}}-\frac{S^{i}}{f_{F}^{3}}+T^{i} \\
+m_{F}^{i}+q_{F}^{i}+\lambda_{F}\left(N_{F}^{i}+B_{F}-B_{F}^{i}\right)
\end{gathered}
$$

where $\mathrm{i}$ is the satellite index and $\mathrm{F}$ represents the index of the GNSS frequency. For GPS satellites, $F=1\left(\mathrm{GPS} \mathrm{L}_{1}\right)$ and $\mathrm{F}=2\left(\mathrm{GPS} \mathrm{L}_{2}\right)$. For GLO satellites $\mathrm{F}=1\left(\mathrm{GLO} \mathrm{L}_{1}\right)$ and $\mathrm{F}=2$ $(\mathrm{GLO} \mathrm{L})$ with

$$
\begin{aligned}
& f_{k L 1}=f_{0 L 1}+k \Delta f_{L 1} \\
& f_{k L 2}=f_{0 L 2}+k \Delta f_{L 2}
\end{aligned}
$$

Here, $k$ represents the frequency channel: $f_{0 L 1}=1602 \mathrm{MHz}$ for GLONASS $\mathrm{L}_{1}$ band, $\Delta f_{L 1}=562.5 \mathrm{kHz}$ frequency separation between the GLONASS carriers in the L1 band, $f_{0 L 2}=1246 \mathrm{MHz}$ for GLONASS $\mathrm{L}_{2}$ band, and $\Delta f_{L 2}=437.5$ $\mathrm{kHz}$ frequency separation between the GLONASS carriers in the $\mathrm{L}_{2}$ band. In the above, $e^{i}$ represents the geometric distance from the receiver to the satellite, $c \delta_{r-c o d e}$ is the receiver clock offset for code, $c \delta_{r \text {-phase }}$ is the receiver clock offset for phase, $c \delta^{i}$ is the satellite clock offset, $I^{i}$ is the first-order ionospheric bias term, $S^{i}$ is the second-order ionospheric bias term, $f_{F}$ is the GNSS frequency, $T^{i}$ is the tropospheric bias. $M_{F}^{i}$ is the multipath error for pseudorange, $m_{F}^{i}$ is the multipath error for carrier-phase, $Q_{F}^{i}$ is the noise for the pseudorange, $q_{F}^{i}$ is the noise for the carrier-phase. bias $_{P, F}$ is the receiver code bias for pseudorange, bias $_{P, F}^{i}$ is the satellite code bias for pseudorange,

$\lambda_{F}$ is the wavelength, $N_{F}^{i}$ is the carrier phase ambiguity term, $B_{F}$ is the receiver fractional cycle bias $(\mathrm{FCB})$, and $B_{F}^{i}$ is the satellite FCB.

For all the PPP daily solutions, a decoupled receiver clock (separate clocks for code and carrier) is applied for both GPS and GLO [10], and the ionosphere-free observable is used without applying any second-order ionospheric bias corrections. The ionospheric-free combinations for the code and phase observables follow the process described by Dach, et al. [11]. The processing settings for the PPP solutions are summarized as follows.

In terms of the troposphere, the hydrostatic component of the zenith total delay is modelled using Saastamoinen [12] and the wet component of the zenith total delay is estimated as a state in the Kalman filter for every observational epoch, i.e. at 30 second intervals. In order to describe how the slant tropospheric delay varies with respect to the receiver-tosatellite elevation angle, the Niell Mapping function [13] or NMF is used. The azimuthal inhomogeneity of the troposphere is also taken into account by estimating two states for the tropospheric gradient $(\mathrm{E}, \mathrm{N})$ using the Chen model [14] and the Chen mapping function [15] to map the tropospheric gradient into the range domain. The Differential Code Bias (DCB) between $\mathrm{C}_{1}$ and $\mathrm{P}_{1}$, are corrected using the products from the Centre for Orbit Determination in European (CODE) [11]. In order to investigate the actual performance of the individual satellite systems, as well as the effect of different products, no weighting functions are applied to the observations, except for the measurement noise standard deviations that are needed for the EKF. These are set to $2.0 \mathrm{~m}$ for pseudo-range measurements, and $0.01 \mathrm{~m}$ for carrier phase measurements, for both GPS and GLONASS.

In addition, for all of the PPP daily solutions: the EMX, ESA and GFZ final precise ephemeris products are used as the input of the satellite coordinates and the satellite clock corrections. Satellite and receiver antenna phase centre offsets and variations are corrected using an Antenna Exchange Format (ANTEX) [16] file from the IGS, i.e. the I08.ATX file which is consistent with what was used in the creation of the precise ephemerides. Periodic deformations of the Earth's crust as solid Earth tides and ocean tidal loading are taken into account following Kouba [16]. The phase wind up correction is also applied in accordance with $\mathrm{Wu}$, et al. [17]. Sub-daily pole and nutation motions are corrected, according to the IERS conventions [18]. And lastly, for all of the PPP daily solutions the carrier phase ambiguities were not fixed to integers, but kept as float, and the cycle slip detection method that has been implemented follows Liu [19]. 


\section{B. Global DD GPS Daily Solution Methodology}

For the UK stations, the processing strategy for the global DD GPS daily solutions is summarized in Table 3. Approximately 150 continuous GNSS stations (CGNSS) in the British Isles, including 100+ that are part of the Ordnance Survey of Great Britain (OSGB) national network, were included in the processing along with some 200+ IGS stations.

Table 1 The processing parameters for Double Difference solutions.

\begin{tabular}{|c|c|}
\hline Software & $\begin{array}{c}\text { Bernese GNSS Software version } \\
5.2[11]\end{array}$ \\
\hline $\begin{array}{c}\text { Products (precise satellite coordinates } \\
\text { and satellite clock offsets) }\end{array}$ & $\begin{array}{c}\text { C13 (CODE repro2/repro_2013) } \\
\text { re-analyzed satellite orbit and } \\
\text { earth orientation parameter } \\
\text { products }\end{array}$ \\
\hline $\begin{array}{c}\text { Satellite and receiver antenna phase } \\
\text { center offsets and variations }\end{array}$ & $\begin{array}{c}\text { I08.ATX models for antenna } \\
\text { phase center variations }\end{array}$ \\
\hline Troposphere & $\begin{array}{c}\text { a-priori modeling of troposphere } \\
\text { effects using VMF1G and } \\
\text { estimation using zenith path } \\
\text { delay and gradient parameters. }\end{array}$ \\
\hline Ionosphere & $\begin{array}{c}\text { mitigation of the first- and } \\
\text { higher-order (second- and third- } \\
\text { order and ray bending) } \\
\text { ionospheric effects }\end{array}$ \\
\hline $\begin{array}{c}\text { Solid earth tides, Ocean tidal loading, } \\
\text { and Atmospheric tidal loading }\end{array}$ & Applied \\
\hline Carrier phase ambiguities & Fixed ambiguity. \\
\hline
\end{tabular}

A total of approximately 150 continuous GNSS stations (CGNSS) in the British Isles, including 100+ that are part of the Ordnance Survey of Great Britain (OSGB) national network, were included in the processing along with some 200+ IGS stations, as illustrated in Fig. 1.

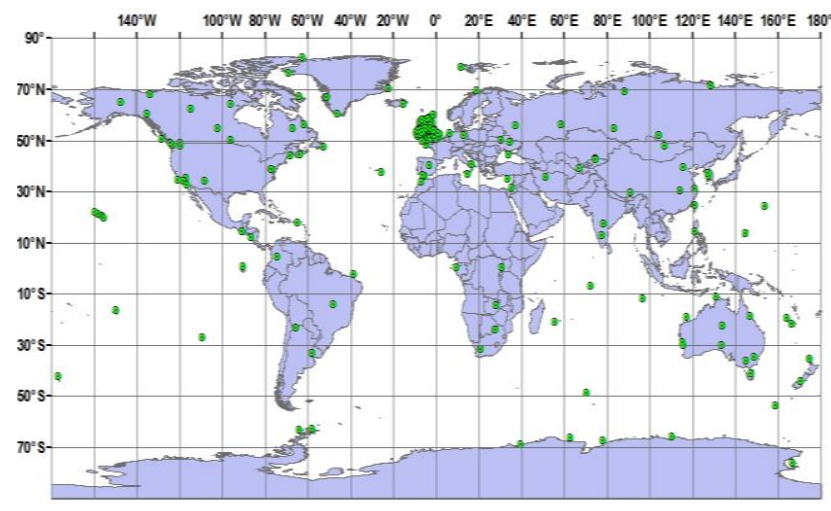

Fig. 1 The network used for the global DD GPS daily solution on $12 / 01 / 2014$.

\section{EXPERIMENTAL RESULTS}

To allow a rigorous testing of the PPP daily solutions, the POINT software, and a thorough investigation of the stability of the precise ephemeris products it was necessary to process a reasonably long-term data set. For the purposes of this study, a data set focussing on the 100+ OSGB CGNSS stations that have daily RINEX observation data files archived as part of BIGF, and were included in the global DD GPS daily solutions created by BIGF, was chosen, with the CGNSS station locations illustrated in Fig. 2.

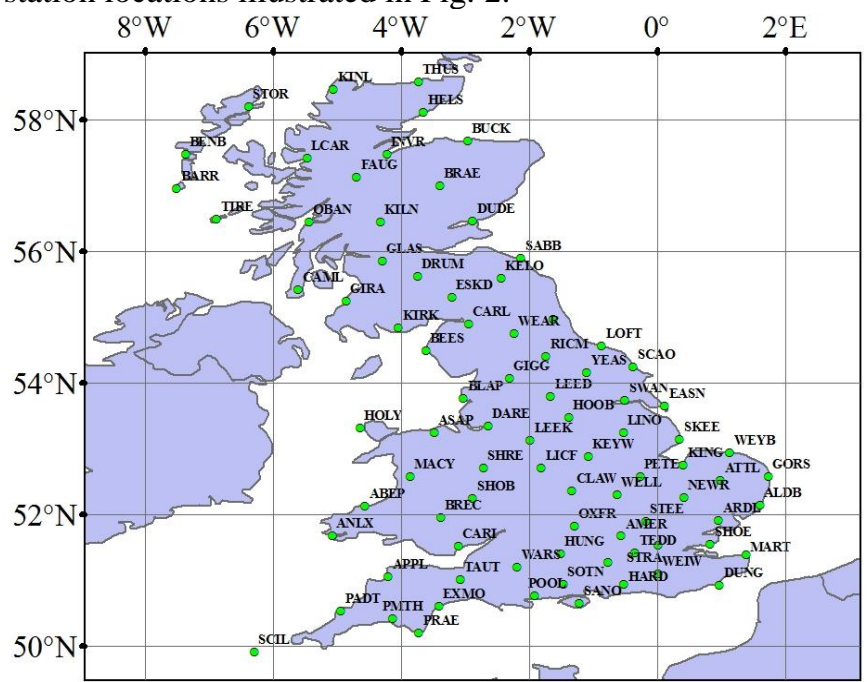

Fig. 2 The 100+ OSGB CGNSS stations included in BIGF that were used for the assessment of PPP in this study

An investigation was carried out based on all 100+ OSGB CGNSS stations (see Fig. 2) over a 7-week period, as detailed in Table 1. In this investigation, only OSGB CGNSS stations that were continuous for a specific GPS week, had optimal 24 hour observations recorded each day, and were also present in the global DD daily solutions were included, which led to data sets with between 56 and 85 OSGB CGNSS stations per week being available for analysis, as also detailed in Table 2 . It is also worth noting that the 56 stations available in GPS week 1775 were also included in all the 7 weeks.

Table 2 GPS week and the number of OSGB CGNSS stations considered in the analysis for each week

\begin{tabular}{|c|c|c|c|c|c|c|c|}
\hline $\begin{array}{c}\text { GPS } \\
\text { Week }\end{array}$ & 1775 & 1776 & 1777 & 1778 & 1783 & 1784 & 1785 \\
\hline $\begin{array}{c}\text { No. of } \\
\text { CGNSS } \\
\text { Stations }\end{array}$ & 56 & 85 & 79 & 74 & 74 & 76 & 79 \\
\hline
\end{tabular}

\section{1) Reference station time-base}

PPP time is relying on the satellites clock offset. However, a time component that is the estimated receiver offsets have to be estimated for the receiver part, many unmodeled errors or offsets will be absorbed by the estimated receiver clock offsets.

Starting with the satellite clock, and more specific with GPS constellation, all the ACs produced GPS precise ephemeris. To look at the actual estimated satellites clocks offset by different AC, Fig. 3 (upper) is an example for GPS 
PRN no. 5 and 7 on DOY 12, 2014 from the three ACs (EMX, ESA and GFZ). It represents the differences from the mean as well as the differences from the mean of the estimated receiver clock offset of ALDB station.
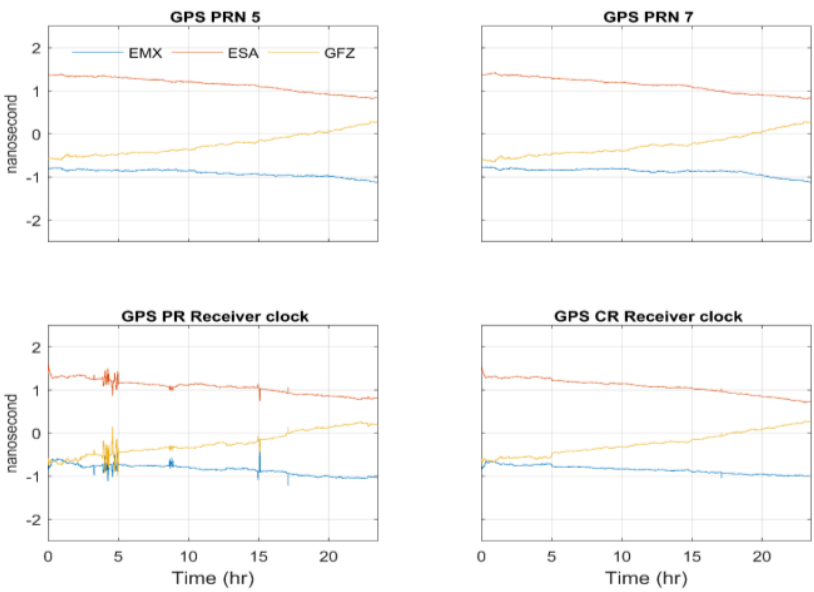

Fig. 3 GPS satellites clock offset differences between EMX, ESA and GFZ(upper), receiver clock offsets differences from the mean for ALDB station (lower), DOY 12, 2014

It is clear from Fig. 3 that there is an offset between the precise satellites clocks from different ACs. This difference is in nanosecond, where every nanosecond multiplied by the speed of light will represent around $30 \mathrm{~cm}$ in the range. Those ACs have different strategy for producing their products. However, all those agencies are following the same part of strategy for the time referencing station, meaning that one reference station from the chosen network (or the IGS network) for that day will be chosen and the base time network will be fixed relative to a specific epoch of the chosen reference station. Even though this reference station has an external frequency instruments, it could have a drift or offsets from the GPS time as can be seen from Fig. 3. This effect will be propagated to the estimated satellites clocks, which is noticed that this offset is almost the same for all satellites.

The propagated time offset into the satellites clock offset will affect the PPP solution. More specifically, it will affect the responsible parameter for balancing the time offset. This parameter is the receiver clock offset. It will absorb the combination of the propagated time offset from all satellites time offset on that specific epoch. In addition, it will absorb the offset propagated in all satellites to be represented as an AC time offset from the truth GPS time. However, it will be absorbed for making the needed balance if it was modelled correctly. This means that if a constrained was made on the receiver clock offset(s), then it will lose the ability to overcome that offsets which will results as an effect on other estimated parameters from PPP. As was showing in Fig. 3 which is an example of the differences from the mean between the estimated receiver clock offsets for both the code and carrier receiver clock offset which clearly showed that the receiver clock offset absorbed the offset caused by the station reference time based.

It is worth mentioned that, the resulted offsets from the reference station time based on the GLO satellites clock offset is not the same for the GLO constellation using the same organization GLO products. Fig. 4 shows an example for GLO satellites no. 1 and 2 for DOY 12, 2014 and the receiver clock offsets from PPP GLO solution for the same station (ALDB) that had been used for PPP GPS.
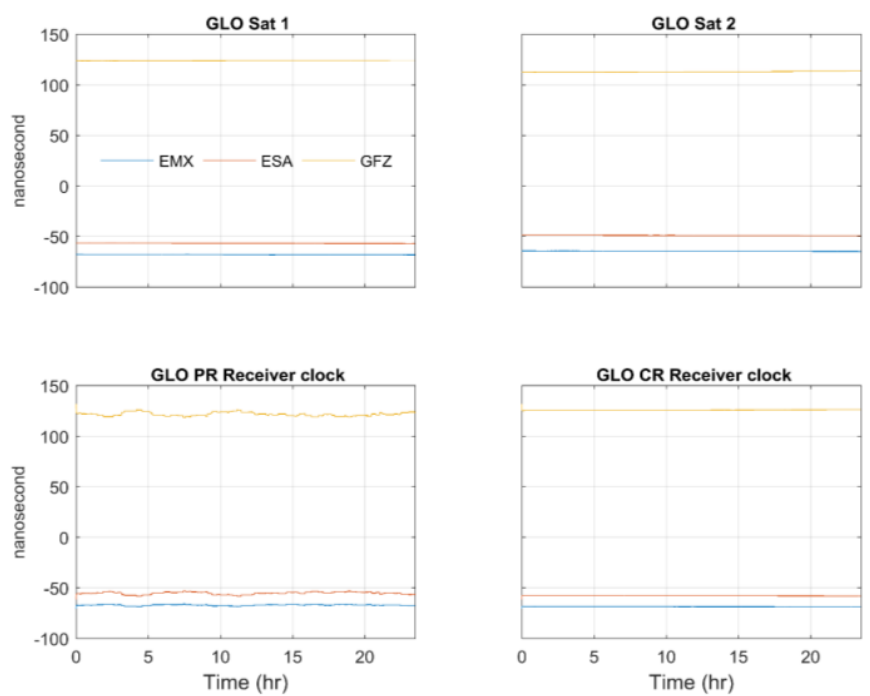

Fig. 4 GLO satellites clock offset differences from the mean between EMX ESA and GFZ, b) receiver clock offsets differences from the mean for ALDB station, DOY 12, 2014

The reference station offset is much higher for the GLO than GPS constellation. Cai and Gao [4] claimed that the difference between the receiver clock offsets for GPS and GLO represents the time difference between the two constellations. This assumption is not entirely accurate. To see why, there is a need to look at the difference between the receiver clock offsets for the same station. In the presented strategy, two different clocks, one carrier and the second is the code clock for every constellation, were estimated. Fig. 5 represents the difference between the estimated receiver clocks offset between GPS and GLO, using all three products (EMX, ESA and GFZ).
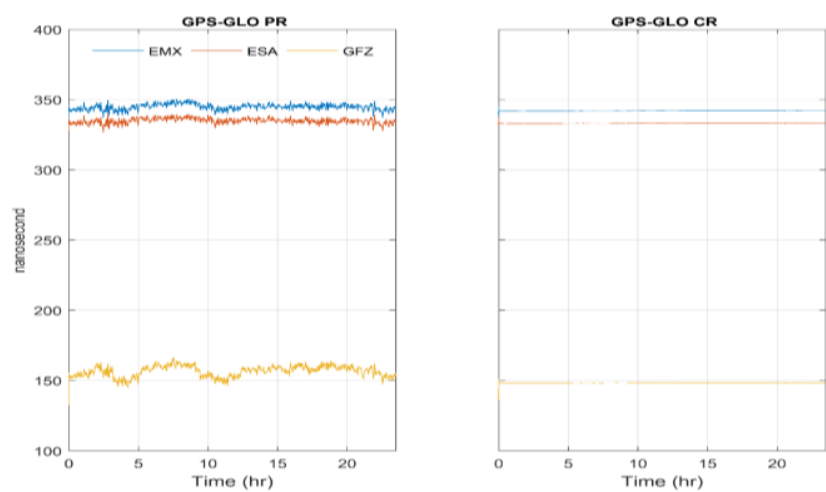

Fig. 5 Receiver clock offset differences between PPP GPS and PPP GLO for EMX, ESA and GFZ for ALDB station DOY 12, 2014

If the assumption from Cai and Gao [4] that the difference between the receiver clock offsets represents the time difference between GPS and GLO is correct then those difference have to be similar for all products type. The 
difference between the estimated receiver clock offsets will be contaminated by the ACs reference station bias as well as the system time difference. It is also evident that the code clock have higher variation and this because of the noise in the code observable, more specifically GLO code.

2) Precise Satellites orbit effect on PPP position estimation

Each AC uses different strategy for estimating the final satellites coordinates, e.g. tropospheric models, tropospheric strategy, as well as different versions of IERS that they used. Therefore, it is important to investigate the level of those differences from the estimated coordinates of satellites. Fig. 6 represents the difference from the mean between the three organizations EMX, ESA and GFZ for the three satellites Cartesian coordinates, on DOY 12, 2014.
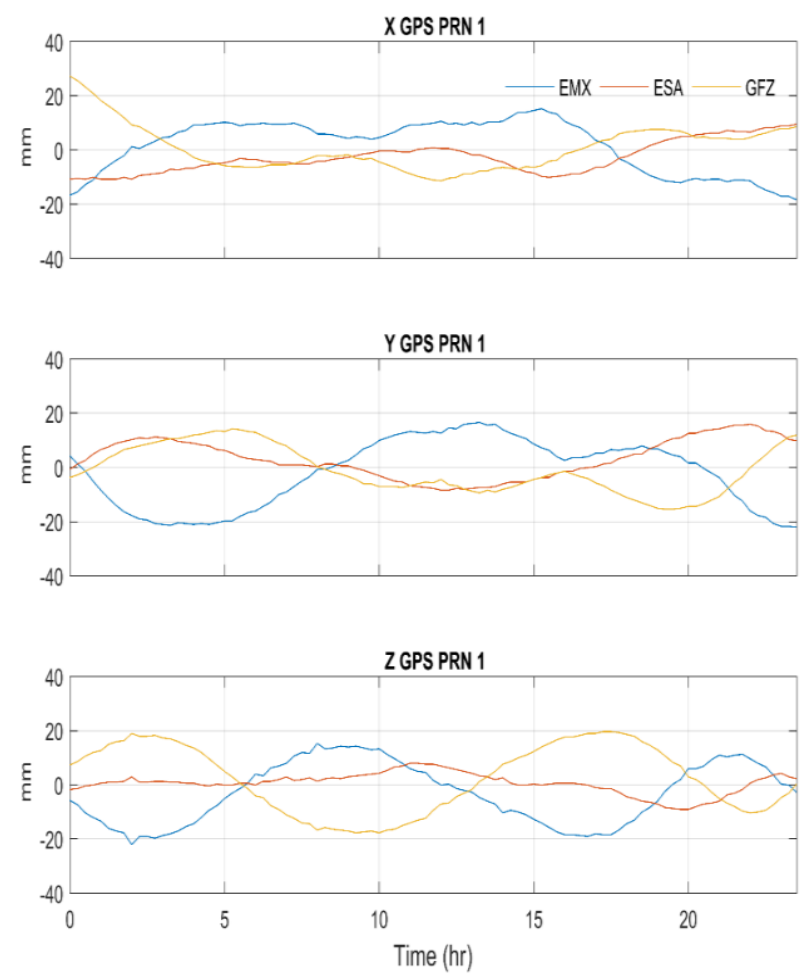

Fig. 6 Differences from the mean between satellites precise coordinates for (EMX, ESA and GFZ) from their mean for DOY 12, 2014

The SP3 files (satellites coordinates) are representing the fundamental parameters for PPP solution because it is the responsible parameters for defining the system coordinates of PPP. The precise coordinates have to be implemented in the design matrix with respect to the receiver coordinates component as known values. Therefore, any differences or small error between the used coordinates and the 'truth' value will theoretically be propagated into the receiver position components, as in:

$$
\frac{\left(\left(X_{ \pm} \text {error }\right)^{s}-X_{r}\right)}{\rho}, \quad \frac{\left((Y \pm \text { error })^{s}-Y_{r}\right)}{\rho}, \frac{\left((Z \pm \text { error })^{s}-Z_{r}\right)}{\rho}
$$

The use of the SP3 files from a specific AC, will give a different solution because even though the difference between the satellite coordinates from different ACs are within centimetres level, and this will affect the receiver position components at the millimetres level. This is because of the adjustment that has to be done using the EKF in this research and the position is in static mode.

Consequently, the effect of using different products from different ACs on the receiver position components can be seen from Fig. 7, which represents the differences between the estimated receiver Cartesian coordinates using the three ACs products and the mean solution for PPP GPS and PPP GLO of ALDB station on DOY 12, 2014.
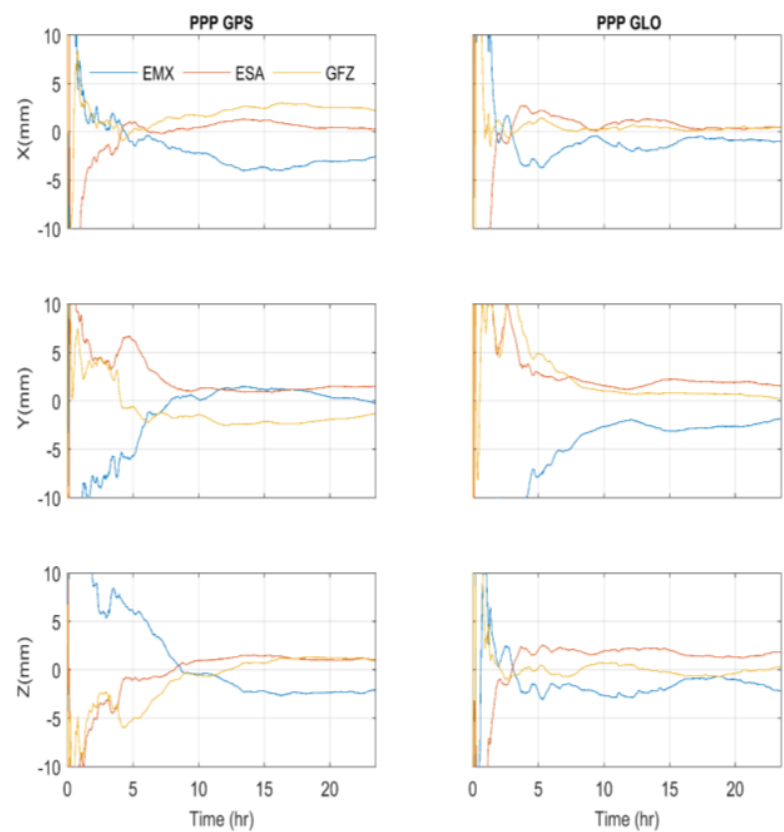

Fig. 7 The effect of using different Products on the Position estimation from PPP GPS

While the processing strategy is the same for all PPP scenarios except the products type, it is clear that the differences between these solutions are come from the products type effect, more specifically, from the different satellites coordinates.

3) Products Effect on the accuracy and precision of PPP

The analysis in terms of repeatability considered weekly solution coordinate estimates as opposed to daily solution coordinate estimates for the stations mentioned in Table 2 with their relevant GPS week. In these instances, for each of the three PPP daily solutions (GPS, GLO and GPS+GLO) and the global DD GPS daily solution, the mean of the daily solution coordinate estimates for seven weeks were calculated. The weekly solution coordinate estimates for each week were then subtracted from the mean to create weekly coordinate differences in terms of Easting, Northing and Up components. Following this, it was possible to use the weekly coordinate differences in order to calculate the repeatability of the weekly solution coordinate estimates in each case in terms of Easting, Northing and Up components. This was done for all the three products (EMX, ESA and GFZ). Bar charts representing the RMS of the repeatabilities of the weekly solution coordinate estimates from PPP (GPS, GLO and 
GPS+GLO) and global DD GPS as individual coordinate components (Plan, Easting, Northing, and Up) for all of the CGNSS stations considered as well as the three products type (EMX, ESA and GFZ) are presented in Fig. 8.

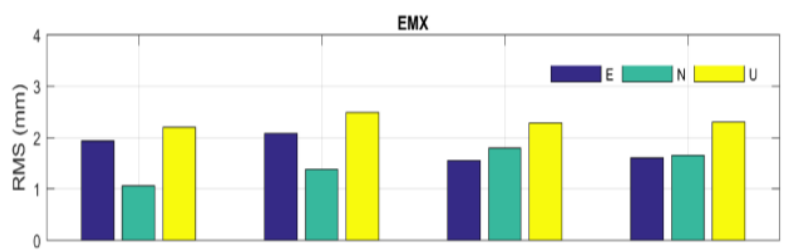

ESA

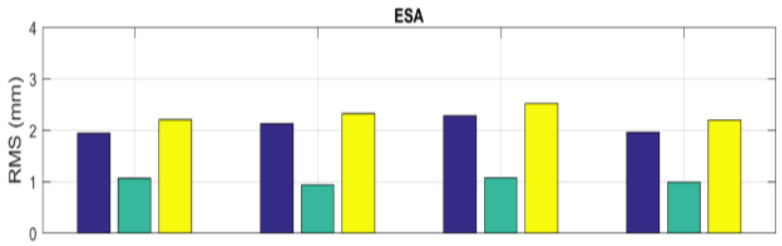

GFZ

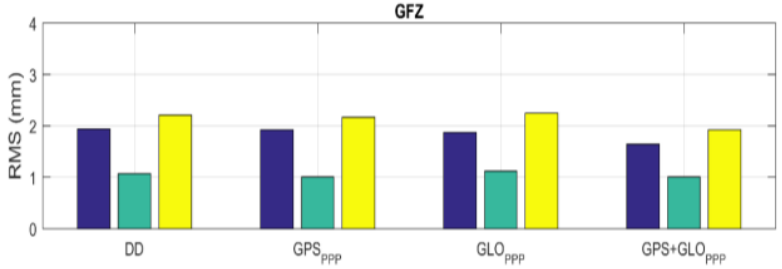

Fig. 8 RMS of the repeatabilities of the weekly solution coordinate estimates from PPP (GPS, GLO and GPS+GLO) and global DD GPS for all CGNSS stations considered using three products(EMX, ESA and GFZ)

Looking at the individual component, the two components that have similar performance for the Northing and Easting are ESA and GFZ. The Northing component in ESA and GFZ has almost similar value and similar stability over time. While the Easting component has a lower performance to being twice the northing values which is the effect of the ambiguity.

Regarding the height component, the precision values almost the same for all PPP scenarios as well as all the used products except the PPP GLO when using GFZ products, see Fig. 6. This means that the GFZ products $(0.8 \mathrm{~mm}$ worse than ESA and $1.3 \mathrm{~mm}$ worse than EMX).

In addition to repeatability, it is also possible to estimate any differences between the weekly solution coordinate estimates from PPP (GPS, GLO and GPS+GLO) and the weekly solution coordinate estimates from global DD GPS. In this regard, measures of accuracy are obtained if the weekly solution coordinate estimates from global DD GPS, which are independent of the weekly solution coordinate estimates from PPP, are assumed to represent the most probable values. Bar charts representing the RMS of the differences between the weekly solution coordinate estimates from PPP (GPS, GLO and GPS+GLO) and the weekly solution coordinate estimates from global DD GPS as individual coordinate components (Easting, Northing, and Up) for all of the CGNSS stations considered as well as the three products type (EMX, ESA and GFZ) are presented in Fig. 9.
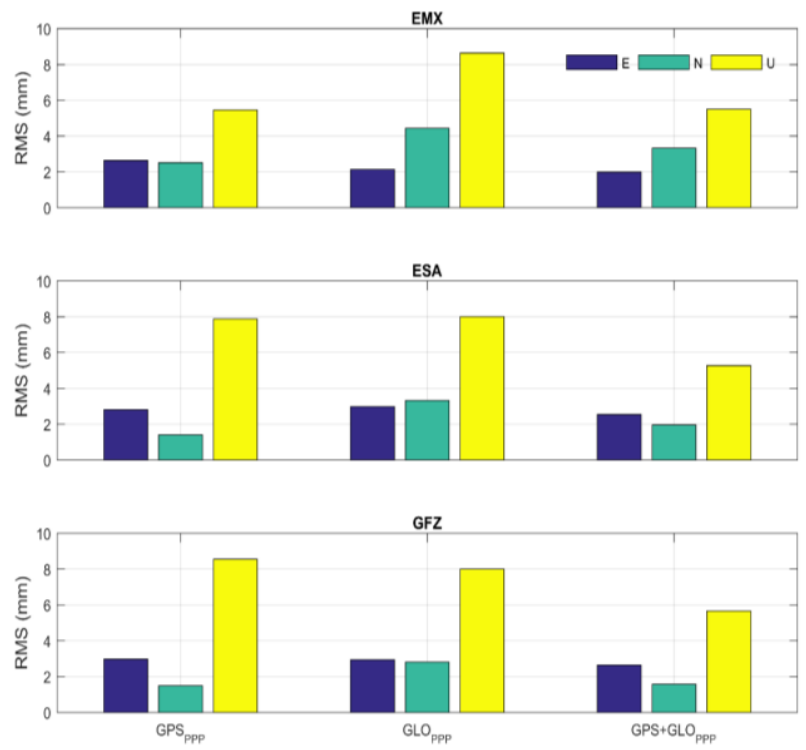

Fig. 9 RMS of the differences between the weekly solution coordinate estimates from PPP (GPS, GLO and GPS+GLO)

To give a comprehensive explanation of any biases present, bar charts representing the mean of the differences, or the biases, between the daily solution coordinate estimates from PPP (GPS, GLO and GPS+GLO) and the weekly solution coordinate estimates from global DD GPS as individual coordinate components (Easting, Northing, and Up) for all of the CGNSS stations considered as well as the three products type (EMX, ESA and GFZ) are presented in Fig. 10.
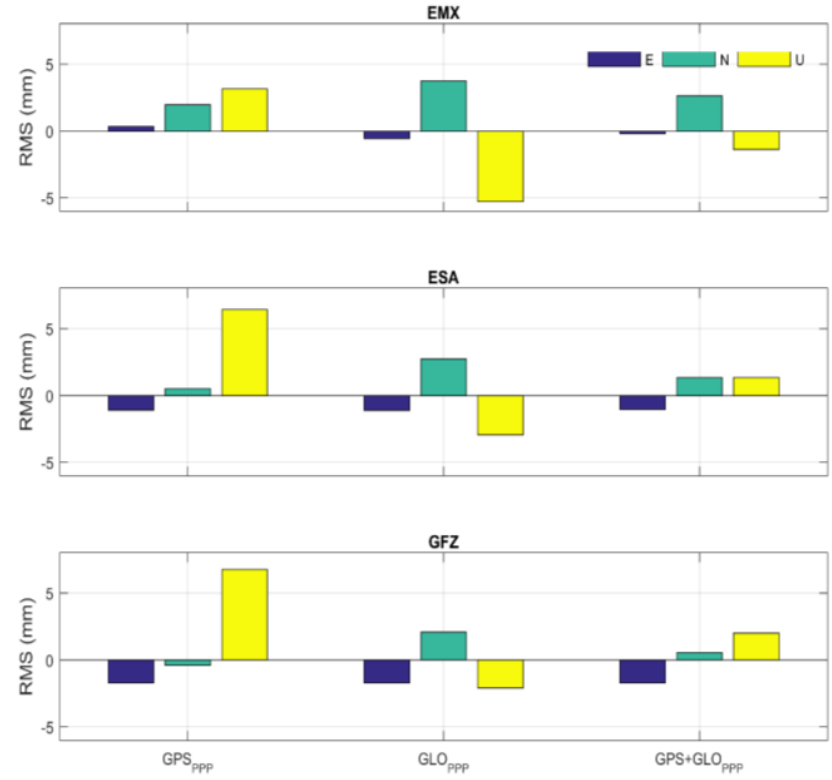

Fig. 10 Mean of the differences, or the biases, between the weekly solution coordinate estimates from PPP (GPS, GLO and GPS+GLO) and the weekly solution coordinate estimates from global DD GPS for all CGNSS stations considered 
It is clear from Fig. 10 that the mean of the differences, or the biases, between the daily solution coordinate estimates from PPP (GPS, GLO and GPS+GLO) and the daily solution coordinate estimates from global DD GPS is a constellation type dependent. In addition, there is a consistently larger bias in the Northing component than in the Easting component, with the Northing component from PPP (GPS, GLO and GPS+GLO) being to the north of the Northing component from global DD GPS. One reason for this could be a reference frame issue, as while the PPP technique relies only on the precise ephemeris products, the DD technique relies on these but is also affected by the network of global CGNSS stations that were used.

Re-considering Fig. 1, which gives an example of the network that was used for the global DD GPS solution on 2014/01/12 (DOY 012 of 2014), the global network contains 97 CGNSS stations to the south of the lowest latitude OSGB CGNSS station (SCIL, lat $=49.9$ ) but only 19 CGNSS stations to the north of the highest latitude OSGB CGNSS station $(\mathrm{KIRW}$, lat $=58.9)$.

Furthermore, the subtle differences in the biases for the Northing coordinate component for PPP GLO and PPP GPS could be a constellation issue related to the higher inclination of the GLONASS satellites when compared to GPS, which results in a significantly different pattern for the GLONASS and GPS constellations as shown in Fig. 11.
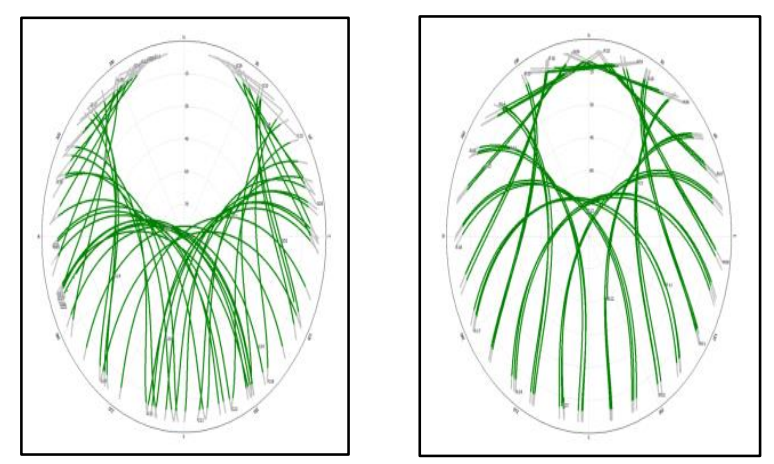

Fig. 10 Representative satellite constellation availability (Left: GPS, Right: GLONASS) for ALDB station on DOY 12, 2014.

To support that idea, the differences between the mean weekly solution of PPP GPS, PPP GLO and PPP GPS+GLO were subtracted from the mean weekly solution from the global DD GPS; see Fig. 12 for 85 stations in GPS week 1776 for all the three products (EMX, ESA and GFZ).

It is clear from the Fig. 12, that all PPP scenarios' Northing differences have almost the same shape and increasing differences with the increasing of the station latitude. These increasing differences indicate the effect of the reduced CGNSS stations to the north for global DD GPS solution. In addition, while PPP GLO has available satellites to the north, it has larger differences than PPP GPS because of the reduced availability of the GPS constellation with the increasing of the station latitude. While PPP GPS+GLO will has the balanced differences between both constellations as was shown in Fig. 12.

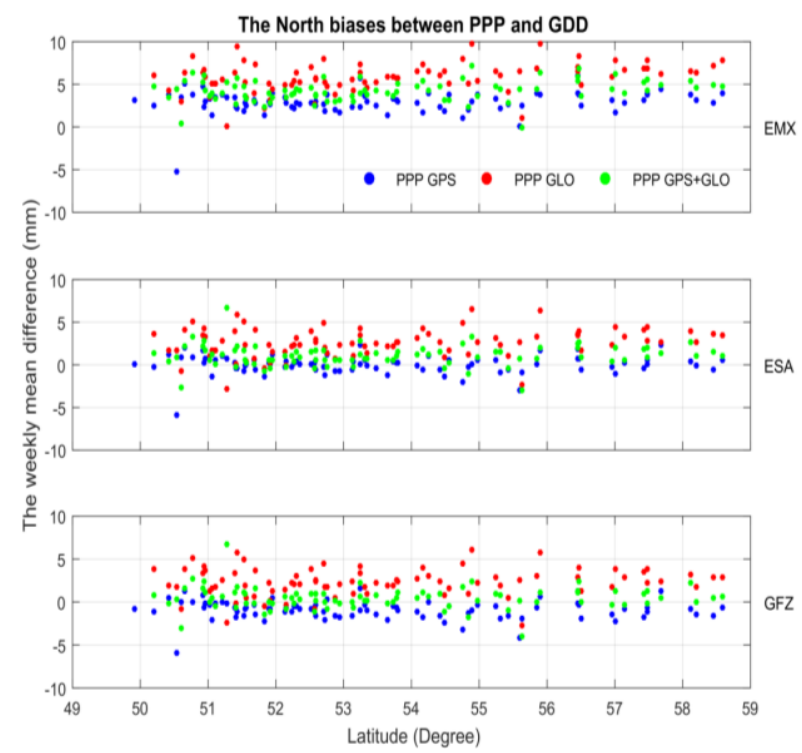

Fig. 11 North biases relative to the GDD Solution for 85 stations in GPS week 1776.

Lastly, considering the biases in the Up coordinate component (Fig. 10), it is clear that in the case of PPP GPS+GLO there is effectively a balancing of the errors present in PPP GPS and PPP GLO. This clearly results in an improvement in the Up component for PPP GPS+GLO over PPP GLO, as the latter may be more affected by any imperfections in the models for GLONASS antenna phase centre variations.

\section{CONCLUSIONS}

In this paper, the effect of the reference station time-base on the precise products has been assessed. It is shown that estimated precise satellite clock are being affected by the chosen reference station depending on the AC. It is also shown that the time-base is being balanced by the receiver clock offsets to be as AC offset type. Further, the effect of the satellites coordinates on the PPP position component has been assessed which is being found to be in the level of millimeters. Furthermore, the effect of these products on the precision and accuracy has been assessed. It is found that the all these products have the same level of precision in the horizontal component because the ACs followed the same strategy over days. It is also found that the Easting component being worse that the Northing component for all products. While for the accuracy part it is found that the products have different behavior especially for the up components. It is concluded that the noticeable bias in the north component is constellation type and latitude dependent. Furthermore, an improvement is found in the Up component for PPP GPS+GLO over PPP GLO, as the latter may be more affected by any imperfections in the models for GLONASS antenna phase centre variations.

\section{ACKNOWLEDGMENT}

The services of the Natural Environment Research Council (NERC) British Isles continuous GNSS Facility (BIGF), www.bigf.ac.uk, in providing archived GNSS data and products to this study, are gratefully acknowledged. Also, 
the first author acknowledges the financial support he received from his government in Iraq during the period of his postgraduate research study at the University of Nottingham. method for a single dual-frequency GPS receiver," Journal of Geodesy, vol. 85, pp. 171-183, Mar 2011.

\section{REFERENCES}

[1] J. F. Zumberge, M. B. Heflin, D. C. Jefferson, M. M. Watkins, and F. H. Webb, "Precise point positioning for the efficient and robust analysis of GPS data from large networks," Journal of Geophysical Research-Solid Earth, vol. 102, pp. 5005-5017, Mar 101997.

[2] K. M. Larson, A. R. Lowry, V. Kostoglodov, W. Hutton, O. Sanchez, K. Hudnut, et al., "Crustal deformation measurements in Guerrero, Mexico," Journal of Geophysical Research-Solid Earth, vol. 109, Apr 282004.

[3] Y. Gao and K. Chen, "Performance analysis of precise point positioning using rea-time orbit and clock products," Positioning, vol. 1, p. 0, 2004.

[4] C. Cai and Y. Gao, "Estimation of GPS-GLONASS System Time Difference with Application to PPP," presented at the Proceedings of the 21st International Technical Meeting of the Satellite Division of The Institute of Navigation (ION GNSS 2008), Savannah, GA, 2008.

[5] N. Reussner and L. Wanninger, "GLONASS Inter-frequency Biases and Their Effects on RTK and PPP Carrier-phase Ambiguity Resolution," presented at the Proceedings of the 24th International Technical Meeting of The Satellite Division of the Institute of Navigation (ION GNSS 2011), Portland, Oregon, 2011.

[6] C. S. Cai and Y. Gao, "Modeling and assessment of combined GPS/GLONASS precise point positioning," Gps Solutions, vol. 17, pp. 223-236, Apr 2013.

[7] F. Guo, X. Li, X. Zhang, and J. Wang, "Assessment of precise orbit and clock products for Galileo, BeiDou, and QZSS from IGS Multi-GNSS Experiment (MGEX)," GPS Solutions, 2016.

[8] J. Mohammed, T. Moore, C. Hill, R. M. Bingley, and D. N. Hansen, "An assessment of static precise point positioning using GPS only, GLONASS only, and GPS plus GLONASS," Measurement, vol. 88, pp. 121-130, 2016.

[9] S. Feng, W. Ochieng, T. Moore, C. Hill, and C. Hide, "Carrier phase-based integrity monitoring for high-accuracy positioning," GPS Solutions, vol. 13, pp. 13-22, 2008.

[10] P. Collins, S. Bisnath, F. Lahaye, and P. HÉRoux, "Undifferenced GPS Ambiguity Resolution Using the Decoupled Clock Model and Ambiguity Datum Fixing," Navigation, vol. 57, pp. 123-135, 2010

[11] R. Dach, U. Hugentobler, P. Fridez, and M. Meindl, "Bernese GPS software version 5.0 (user manual of the Bernese GPS software version 5.0)," 2007.

[12] J. Saastamoinen, "Contributions to the theory of atmospheric refraction," Bulletin Géodésique, vol. 107, pp. 13-34, 1973.

[13] A. E. Niell, "Global mapping functions for the atmosphere delay at radio wavelengths," Journal of Geophysical Research-Solid Earth, vol. 101, pp. 3227-3246, Feb 101996.

[14] M. Meindl, S. Schaer, U. Hugentobler, and G. Beutler, "Tropospheric gradient estimation at CODE: Results from global solutions," Journal of the Meteorological Society of Japan, vol. 82, pp. 331-338, Mar 2004

[15] G. Chen and T. A. Herring, "Effects of atmospheric azimuthal asymmetry on the analysis of space geodetic data," Journal of Geophysical Research-Solid Earth, vol. 102, pp. 20489-20502, Sep 101997.

[16] J. Kouba. (2009). A guide to using International GNSS Service (IGS) products. Available: ftp://ftp.igs.org/pub/resource/pubs/UsingIGSProductsVer21.pdf

[17] J. Wu, S. Wu, G. Hajj, W. Bertiger, and S. Lichten, "Effects of antenna orientation on GPS carrier phase," Manuscripta Geodaetica, vol. 18, pp. 91-98, 1993.

[18] D. D. McCarthy and G. Petit, "IERS conventions (2003)," DTIC Document, Germany2004. 